\title{
Assessment of Rapid Antigen Detection Testing in Diagnosing Group A Streptococcal Pharyngitis in Comparison with Gold Standard Bacterial Culture
}

\author{
M. Nagaraja Gurumoorthy and G. Manjula*
}

Institute of Microbiology, Madurai Medical College, Madurai, India

Chief Civil Surgeon, Govt. Hospital, Balarengapuram, Madurai

*Corresponding author

\section{Keywords}

Acute pharyngitis,

Group A

Streptococci, RADT

\section{Article Info}

Accepted:

20 July 2019

Available Online:

10 August 2019

\section{A B S T R A C T}

Acute pharyngitis is a very common illness that can be caused by different viruses and bacteria, Group A streptococci (GAS) being the most common bacterial cause. However, clinical examination cannot be used to differentiate viral and group A strep pharyngitis, even for experienced clinicians. Objectives of the study is to determine the impact of rapid antigen detection testing (RADT) to identify group A beta haemolytic Streptococcus in acute pharyngitis and to compare the RADT with throat swab culture in diagnosing acute GAS pharyngitis. Throat swabs were collected from patients with sore throat. All the samples were tested immediately by Rapid antigen detection test and also cultured on to Blood agar plate for isolation of Group A streptococci. The effectiveness of RADT was analysed against the gold standard culture. Out of 102 samples collected 29 were positive for Streptococcal infection by the gold standard culture, showing a prevalence of $28.4 \%$ in symptomatic patients. Of this 29 culture positive cases, RADT was positive for Streptococcal antigen for 20 cases with a sensitivity of $69 \%$. The prevalence of Streptococcal pharyngitis is only $28.4 \%$ in clinically suspected cases of 5-15 years age group, the majority of the other cases must be due to viral etiology. Hence it is necessary to screen all the clinically suspected cases for GAS, so that unnecessary antibiotic prescription can be avoided. RADT can be used as a screening test though the sensitivity of RADT is $69 \%$.

\section{Introduction}

Acute pharyngitis is a very common illness that can be caused by different viruses and bacteria. Group A streptococci (GAS), the most common bacterial cause, is estimated to cause $20 \%$ to $30 \%$ of pharyngitis episodes in children and approximately $5 \%$ to $15 \%$ of pharyngitis infections in adults ${ }^{(1)}$. Group A streptococcus (GAS) is one of the most common and important pathogens causing community-associated infections. (2) The epidemiological data of disease due to GAS from developing countries are scarce. In 
India, prevalence of GAS pharyngitis and its carriage estimated previously varies from 4.2$13.7 \%$ and $11.2-34 \%$, respectively. ${ }^{(3),(4)}$

Group A strep pharyngitis can occur in people of all ages, but it is most common among children 5 - 15 years of age. The infection spreads from person to person through direct contact, through saliva or nasal secretions. The transmission is more in crowded conditions like school,day care centre etc.The infection presents with sudden onset of sore throat, odynophagia and fever. Viral pharyngitis resolves without sequelae but GAS pharyngitis has well proved complications of development of Rheumatic fever and Glomerulonephritis.

The presenting complaints and mode of spread is similar for viral and GAS pharyngitis. However, clinical examination cannot be used to differentiate viral and GAS pharyngitis, even for experienced clinicians. Treating a person with viral pharyngitis with an antibiotic is totally unnecessary. At the same time, treating a person with Group A Strep pharyngitis with an appropriate antibiotic for 24 hours or longer generally shortens the duration of symptoms, reduce risk of developing sequelae (acute rheumatic fever) and also eliminates their ability to transmit the bacteria to family members, classmates, and other close contacts ${ }^{(5) .}$

Hence it is necessary to diagnose GAS pharyngitis to decide whether antibiotic has to be started or not.

Throat swab culture on a sheep blood agar plate (BAP), as first described by Breese and Disney in 1954 (6), was the accepted standard technique for the diagnosis of GAS pharyngitis for nearly five decades (7). The major disadvantage of culturing throat swabs on BAPs is the delay (overnight or longer) in obtaining the results. Commercial rapid antigen detection tests (RADTs) were developed in the early 1980s, for the rapid identification of GAS directly from throat swabs.

Hence, the diagnosis of group A strep pharyngitis can be made by either a rapid antigen detection test (RADT) or a throat culture, Throat culture being the gold standard diagnostic test. Though RADT will give an immediate result, the regular use of this by clinician is very low.

In this backround this study aims at comparing the effectiveness of a rapid antigen detection test against throat swab culture in diagnosing acute GAS pharyngitis.

The main objectives of this study, to determine the impact of rapid antigen detection testing (RADT) in identifying group A beta haemolytic Streptococcus in acute pharyngitis. And also to compare the RADT with throat swab culture in diagnosing acute GAS pharyngitis.

\section{Materials and Methods}

This study was conducted after getting the Institutional ethical committee clearance.

Sample size: 102 throat swab specimen.

Inclusion criteria: Patients with sore throat or with a diagnosis of pharyngitis, in the age group of 5-15 years.

Exclusion criteria: Patients with sore throat of age 5-15 years.

Study centre: Tertiary care hospital, Tamilnadu.

Study period: Six months

Throat swabs were collected from patients seeking medical care because of a sore throat 
or with a diagnosis of pharyngitis, after getting their consent.

Basic demographic details of the patients were collected before taking throat swab.

Two throat swabs were collected from each patient following standard precautions

Swabs were collected from posterior pharyngeal wall and both tonsils without touching other areas of the oropharynx

All the samples were tested immediately by the Rapid antigen detection test (Antigen extraction and Immuno chromate graphy) as per the protocol given in the kit and the results were recorded.

As a comparison, all the samples were cultured on to Blood agar plate for isolation of Group A streptococci and incubated at $37^{\circ} \mathrm{C}$ in a candle jar. Blood agar plates showing beta hemolytic colonies with colony morphology suggestive of beta hemolytic streptococcus species were processed further. Screening for GAS was done by bacitracin discs $(0.04 \mathrm{U})$ and sulfamethoxazoletrimethoprim (SXT) susceptibility test. The results of both tests were analyzed to evaluate the effectiveness of RADT against the gold standard culture.

\section{Results and Discussion}

The study group consisted of 102 patients who were clinically diagnosed pharyngitis patients. Among these, 43 (42\%) were males and $59(58 \%)$ were females.

As seen in Table 1, Out of 102 samples collected 29 were positive for Streptococcal infection by the gold standard culture, showing a prevalence of $28.4 \%$ in symptomatic patients.

Of this 29 culture positive cases, RADT was positive for Streptococcal antigen for 20 cases, as seen by Table 2 .

Nine samples were negative for RADT, but positive for Streptococcal infection by the gold standard culture

Sensitivity of RADT is $69 \%$ compared to the gold standard culture.

Table.1 Sex wise distribution of cases by throat swab culture

\begin{tabular}{|l|l|l|l|}
\hline Sex of the patient & Positive & Negative & Total \\
\hline Male & 11 & 32 & 43 \\
\hline Female & 18 & 41 & 59 \\
\hline
\end{tabular}

Table.2 Evaluation of RADT against culture

\begin{tabular}{|l|l|l|}
\hline $\begin{array}{l}\text { RADT } \\
\text { Culture }\end{array}$ & Positive & Negative \\
\hline Positive & 20 & 9 \\
\hline Negative & 0 & 73 \\
\hline
\end{tabular}


Specificity of RADT is $100 \%$ compared to the gold standard culture

In this study, the effectiveness of a rapid antigen detection test against throat swab culture in diagnosing acute GAS pharyngitis was evaluated.

This study shows a prevalence of $28.4 \%$ Streptococcal infection by throat swab culture, which is comparable to the study conducted by Kalpana et al., which showed a prevalence of GAS pharyngitis and tonsillitis $36 \%$ in slum areas ${ }^{(8)}$ in Chennai. Most of the cases in this study were from rural backround. Crowding of person and a compromised living standards may be the reason for the prevalence of this infection in rural areas.

Though the number of female cases are more in this study when compared to male, is not statistically significant. This is similar to the study by Smriti Chauhan et al., ${ }^{(9)}$.

RADT detected 20 out of 29 culture positive cases, having a sensitivity of $69 \%$ which is lesser than in the study conducted by Kose E.et.al,showing $92 \% \quad(10)$ and greater sensitivity than in the study by Susanna Felsenstein et al., in $2014^{(6)}$ which had a sensitivity of $55.2 \%$. This difference in sensitivity may be due to the variation in the kits used. All the RADT positive specimens were positive by blood culture also, which is a gold standard one.So, the specificity of the test is $100 \%$. Both the sensitivity and specificity of this RADT implies that it can be used as a screening test.

The high specificity of rapid antigen detection tests (RADTs) implies that positive results may not require throat culture confirmation and could be used as a basis to prescribe antibiotics in children with pharyngitis ${ }^{(11)}$

The guidelines of the Infectious Diseases
Society of America (IDSA) ${ }^{(7)}$ suggest that swabbing the throat and testing for GABHS pharyngitis by RADT and/or culture should be done because the clinical features alone do not reliably discriminate between GABHS and viral pharyngitis except when overt viral features such as rhinorrhoea, cough exist.

The more important information regarding the diagnostic tests for GAS pharyngitis, both RADT and Culture was negative in $71.5 \%$ (73/102 cases ) of symptomatic patients. This excludes the role of most common bacterial causative agent in pharyngitis cases. And these patients do not require antibiotic prescription. Hence, in the first assessment, RADT results will help the physician to decide regarding prescription of antibiotics in patients with pharyngitis. A similar report was obtained in a study done by Kose E.et.al ${ }^{(10)}$ saying that RADT not only helps to reduce the antibiotic prescription but also reduces the cost of treatment in non-Group A streptococcus pharyngitis. When cost is considered in the management of pharyngitis, RADTs have been shown to be the more costeffective when compared directly with culture, ${ }^{(12)}$ and this is in addition to the advantage of considerably reduced time for accurate diagnosis.

In this study GAS was the cause of pharyngitis in only $28 \%$ of symptomatic patients. This is similar to a study conducted by, Steinman MA et al., where GAS was the cause in only $15-30 \%$ of such cases, but antibiotics prescription was for $55-75 \%$ of these cases. ${ }^{(13) .}$ Most studies indicate that the RADT reduces inappropriate antibiotic prescription for non-GAS pharyngitis ${ }^{(14) .}$

There is also relatively little published information about how physicians in practice actually use RADTs. We also need a direct evidence based study regarding the magnitude of benefit to patients from rapid streptococcal 
testing, comparing the use of tests with the main alternatives, blood culture.

As a result, we can conclude that, in developing countries where unnecessary antibiotic usage is common, performing the RADT for all patients with pharyngitis has an important effect on reducing unnecessary antibiotic prescription, antibiotic costs and possible antibiotic resistance.

RADTs are generally more expensive than BAP cultures, but they have many benefits, such as earlier treatment-within $48 \mathrm{~h}$ after onset - which can provide symptomatic relief, reduce the risk of spread of GAS and enable the patient to return to school or work sooner. Furthermore, the need for follow-up management is lessened, and additional testing can be avoided ${ }^{(15) .}$

Thus, our study showed that the RADT can be used as screening test as an out patirent procedure which can give result within 24 hrs. This will not only decrease the frequency of unnecessary antibiotic prescription in patients with non-GAS pharyngitis, but also increases appropriate antibiotic prescription in patients with GAS pharyngitis.

\section{Outcome}

Acute pharyngitis commonly affects paediatric age group.

All the pharyngitis cases are treated empirically with antibiotics irrespective of causative agent while viral pathogens are the common causative agent.

As Gp A Streptococci is the most common bacterial cause for pharyngitis, a rapid detection method will help to confirm the diagnosis earlier.

The rapid and specific diagnosis of GAS will not only help to initiate specific and prompt treatment to avoid complications and also reduces the unnecessary usage of antibiotics reducing the emergence of drug resistance.

RADT can be used as a point of care test in diagnosing GAS pharyngitis

A strategy in prescribing antibiotics to patients with acute pharyngitis has to be developed

The need for active surveillance to detect prevalence of GAS pharyngitis and the carrier state in the community.

\section{Acknowledgement}

The financial support for this study was given by Tamilnadu State Reasearch Committee, King Institute of Preventive Medicine and Reserarch, Chennai - 600032 for the financial year 2017-18.

\section{References}

1.GroupA Streptococcal (GAS) Disease: https://www.cdc.gov/groupastrep/index

2. Owobu AC, Sadoh WE, Oviawe O. Streptococcal throat carriage in a population of nursery and primary school pupils in Benin City, Nigeria. Niger J Paediatr 2013;40:389-94.

3. Pavan C, Arvind N, Vishrutha KV, Vidyalakshmi K, Shenoy S Surveillance of Group A streptococcal throat infections among school children in Mangalore. Int J Biol Med Res 2013;4:3585-9.

4.Kumar R, Vohra H, Chakraborty A, Sharma YP, Bandhopadhya S, Dhanda V, et al., Epidemiology of group A streptococcal pharyngitis and impetigo: A crosssectional and follow up study in a rural community of northern India. Indian $J$ Med Res 2009;130:765-71. 
5. Brahmadathan $\mathrm{K} \quad \mathrm{N}$, Gladstone $\mathrm{P}$. Microbiological diagnosis of streptococcal pharyngitis: Lacunae and their implications. Indian $\mathrm{J}$ Med Microbiol 2006;24:92-6

6. Susanna Felsenstein, Diala Faddoul, Richard Sposto, Kristine Batoon, Claudia M. Polanco, Jennifer Dien Bard,Molecular and Clinical Diagnosis of Group A Streptococcal Pharyngitis in Children.Journal of Clinical Microbiology Oct 2014, 52 (11) 38843889; DOI: 10.1128/JCM.01489-14

7. Bisno, A. L., M. A. Gerber, J. M. Gwaltney, Jr., E. L. Kaplan, and R. H. Schwartz. 2002. Practice guidelines for the diagnosis and management of group A streptococcal pharyngitis. Infectious Disease Society of America. Clin. Infect. Dis. 15:113-125

8. S.Kalpana1,Jasmine S Sundar, S.Parameshwari1; Isolation and Identification of Group AStreptococcal Infection Among Slum Children in the Age Group of 5-15 Years in Chennai. IOSR Journal of Pharmacy and Biological Sciences (IOSRJPBS). Volume 2, Issue 1(July-August 2012), PP27-30 www.iosrjournals.org

9. Smriti Chauhan, Nitin Kashyap, Anil Kanga, Kamlesh Thakur, Anuradha Sood, Lata Chandel; Genetic Diversity among Group A Streptococcus Isolated from Throats of Healthy and Symptomatic Children, Journal of Tropical Pediatrics, Volume 62, Issue 2, 1 April 2016, Pages 152-157, https://doi.org/10.1093/tropej/fmv092

10. Engin Kose Seda Sirin Kose.2016. The
Effect of Rapid Antigen Detection Test on Antibiotic Prescription Decision of Clinicians and Reducing Antibiotic Costs in Children with Acute PharyngitisJournal of Tropical Pediatrics, Volume 62, Issue 4, 308315. https://doi.org/10.1093/tropej/fmw014

11.Cohen JF, Bertille N, Cohen R, Chalumeau M. Rapid antigen detection test for group A streptococcus in children with pharyngitis. Cochrane Database of Systematic Reviews 2016, Issue 7. Art. No.: CD010502. DOI: 10.1002/14651858.CD010502.pub2.

12.McIsaac WJ, Kellner JD, Aufricht P, Vanjaka A, Low DE. Empirical validation of guidelines for the management of pharyngitis in children and adults. JAMA 2004;291:1587-95.

13.Steinman MA, Gonzales R, Linder JA, et al., Changing use of antibiotics in community-based practice, 1991-1999. Ann Intern Med 2003; 138: 525-33.

14. Maltezou HC, Tsagris V, Antoniadou A, et al., Evaluation of a rapid antigen detection test in the diagnosis of streptococcal pharyngitis in children and its impact on antibiotic prescription. $J$ Antimicrob Chemother 2008;62:140712.

15. American Academy of Pediatrics. Group A streptococcal infections. In: Pickering LK (ed). Red Book: 2003 Report of the Committee on Infectious Diseases. 26th ed. Elk Grove Village, IL: American Academy of Pediatrics, 2003, 573-84.

\section{How to cite this article:}

Nagaraja Gurumoorthy, M. and Manjula, G. 2019. Assessment of Rapid Antigen Detection Testing in Diagnosing Group A Streptococcal Pharyngitis in Comparison with Gold Standard Bacterial Culture. Int.J.Curr.Microbiol.App.Sci. 8(08): 2337-2342. doi: https://doi.org/10.20546/ijcmas.2019.808.270 